\title{
Global convergence of new conjugate gradient method with inexact line search
}

\author{
Chergui Ahmed ${ }^{1}$, Bouali Taher ${ }^{2}$ \\ ${ }^{1}$ Mathematics and Computer Science Department, Laboratory of Mathematics, Informatics and Systems (LAMIS), \\ Tebessa University, Algeria \\ ${ }^{1}$ Department of Mathematics, Khenchela University, Khenchela, Algeria \\ ${ }^{2}$ Department of Mathematics, Faculty of Science, Jazen University, Jazen, Saudi Arabi
}

\begin{tabular}{l}
\hline \hline Article Info \\
\hline Article history: \\
Received Feb 13, 2020 \\
Revised Aug 13, 2020 \\
Accepted Oct 1, 2020
\end{tabular}

Keywords:

Conjugate gradient

Global convergence

Strong wolf line search

Unconstrained optimization

\begin{abstract}
In this paper, We propose a new nonlinear conjugate gradient method (FRA) that satisfies a sufficient descent condition and global convergence under the inexact line search of strong wolf powell. Our numerical experiment shaw the efficiency of the new method in solving a set of problems from the CUTEst package, the proposed new formula gives excellent numerical results at CPU time, number of iterations, number of gradient ratings when compared to WYL, DY, PRP, and FR methods.
\end{abstract}

This is an open access article under the CC BY-SA license.

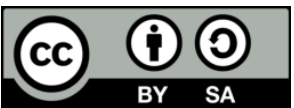

\section{Corresponding Author:}

Chergui Ahmed

Mathematics and Computer Science Department

Laboratory of Mathematics, Informatics and Systems (LAMIS)

Larbi Tebessi University-Tebessa, Algeria

Department of Mathematics, Khenchela University, Khenchela 40004, Algeria

Email: ahmed.chergui@univ-tebessa.dz

\section{INTRODUCTION}

The optimization problem finds application in several fields, such as pure mathematics, mathematical and computational physics, mathematical physics, fluid dynamics, an traffic routing in telecommunication systems [1], cyber-physical security [2], intelligent transportation systems [3], and smart grids [4]. The conjugate gradient method is an effective one for solving large-scale unconstrained optimization problems because it need not the storage of any matrices. Well-known conjugate gradient methods are [5-9]. Global convergence properties of these methods have been studied [9-12].

In this paper, we consider the following unconstrained optimization problem:

$$
(p): \min \{f(x)\}: x \in \underline{\mathrm{R}}^{n}
$$

where $f$ smooth and its gradient $\nabla f\left(x_{k}\right)$ is available

$$
x_{k+1}=x_{k}+t_{k} d_{k}, t_{k}>0 \quad k \quad 0 ; 1 ; 2 ; 3
$$


where $t_{k}$ a positive step size along the search direction obtained by line search. $x_{k}$ is the current iterative point and $d_{k}$ is search direction has the form

$$
\text { By } d_{k}= \begin{cases}-g_{k} & \text { if } k=1 \\ -g_{k}+\beta_{k} d_{k-1} & \text { if } k \geq 2\end{cases}
$$

where $\beta_{k}$ a parameter characterizes the CG method and $g_{k}$ denotes $\nabla f\left(x_{k}\right)$;

The main difference among CG methods is in the formulas of computing their parameters. Some of the well known CG methods are reviewed in [13]. A very famous formula for computing $g_{k}$ is proposed by Fletcher and Reeves (FR) [5] as following

$$
\begin{aligned}
& B_{k}^{F R}=\frac{\left\|g_{k}\right\|^{2}}{\left\|g_{k-1}\right\|^{2}} \text { Fletcher Reeves [5] } \\
& B_{k}^{P R P}=\frac{g_{k}^{T}\left(g_{k}-g_{k-1}\right)}{\left\|g_{k-1}\right\|^{2}} \text { Polak - Ribière - Polyak [6] } \\
& B_{k}^{D Y}=\frac{g_{k}^{T} g_{k}}{\left(\left(g_{k}-g_{k-1}\right)^{T} d_{k-1}\right.} \text { Dai - Yuan [9], } \\
& B_{k}^{W Y L}=\frac{g_{k}^{T} g_{k}-\frac{\left\|g_{k}\right\|}{\left\|g_{k-1}\right\|_{k-1}}}{g_{k-1} g_{k-1}} \text { Wei et al. [10], }
\end{aligned}
$$

where \|. \| Denotes the Euclidean norm. This formula is usually considered the rst nonlinear CG parameter [14]. Having the direction $d_{k}$, the ideal choice for the steplength $t_{k}$ would be the global minimizer of, conditions that require $t_{k}$ satisfying. In order to find the step length $\left(\alpha_{k}\right)$, we use strong wolf powell (SWP) line search,

$$
\begin{aligned}
& f\left(x_{k}+t_{k} d_{k}\right) \leq f\left(x_{k}\right)+\delta t_{k} g_{k}^{T} d_{k} \\
& \left|\nabla f\left(x_{k}+t_{k} d_{k}\right)^{T} \cdot d_{k}\right| \leq-\sigma g_{k}^{T} d_{k}
\end{aligned}
$$

where $\left(0<\delta<\frac{1}{2}\right)$ and $(0<\sigma<1)$

Strong Wolfe conditions used for establishing the global convergence in [9, 12], and [14-17]. The pioneer works about the global convergence of FR method with inexact line search was proposed by Al-Baali [18]. He proved that the FR method satisfied the sufficient descent directions and globally convergent under the (SWP) conditions with $0 \triangleleft \delta \triangleleft \sigma \triangleleft \frac{1}{2}$, in $[9,19]$. This result was extended to $\sigma=\frac{1}{2}$. It is shown that FR method with the (SWP) line search may not be a descent direction for the case that $\sigma>\frac{1}{2}$. For the $B_{k}^{F R}$, neither Armijo nor Wolfe line search, guarantee that the condition suffucient descent. In 2006 [20], Nocedal, J. and Wright, S. (2006) [21-23]. The paper is organized as follows, in section 2. We introduce the new algorithm for $B_{k}$ in section3, we analyze the global convergence property of the new method. Finally, numerical results and conclusion in sections 4 and 5.

\section{NEW ALGORITHM OF FRA}

We propose a new $B_{K}$ for the $C G$ method. The sequence of iteration $x_{k}$ in the new method is obtained from (2) for which the direction $d_{-} \mathrm{k}$ is computed by (3). While the parameter $B_{k}$ parameter $\mathrm{B}_{\mathrm{k}}$ in the new method is;

$$
B_{k}^{F R A}=\lambda \frac{\left\|g_{k}^{T}\right\|^{2}}{\left\|g_{k-1}^{T}\right\|^{2}} \lambda \in(0 ; 1)
$$

where FRA designed the new modified method by Ahmed Chergui.

Note that, for the direction $d_{k}$ defined by (3), withthe CG parameter computed by (10), we have,

$$
g_{k}^{T} d_{k}=-\left\|g_{k}\right\|^{2}+\lambda \frac{\left\|g_{k}^{T}\right\|^{2}}{\left\|g_{k-1}^{T}\right\|^{2}} d_{k-1}^{T} g_{k}
$$


By the Cauchy-Schwarz inequality, it can be concluded that,

$$
g_{k}^{T} d_{k} \leq-\left\|g_{k}\right\|^{2}+\lambda\left\|g_{k}\right\|^{2}=(-1+\lambda)\left\|g_{k}\right\|^{2}<0
$$

So, the new direction $d_{k}$ is satisfied.

In the new CG method, the step $t_{k}$ is determined by the (SWP). To this aim, we use a backtracking approach to compute the steplength. Now we are ready to propose the algorithm of the new CG method (10)

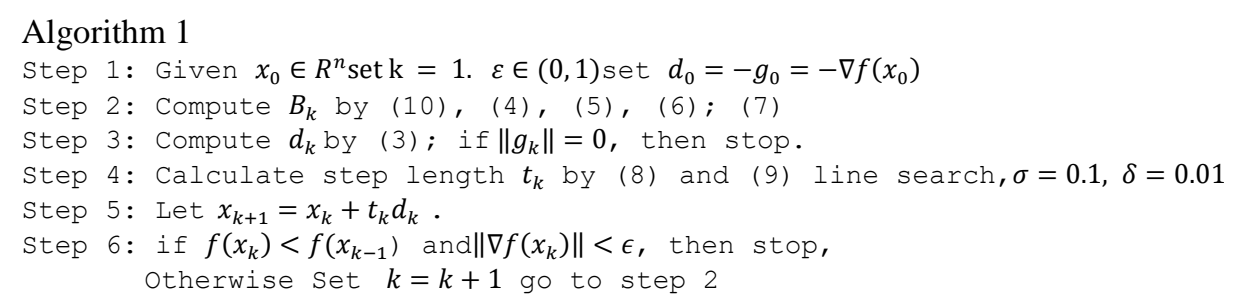

\section{THE GLOBAL CONVERGENCE PROPRIETES} assumption:

In this section, we analyze the convergence of FRA method. To this aim, we made the following Assumption 1

(H1) The objective function $f$ is bounded below on the level set $R^{n}$ and is continuous and differentiable in neighborhood $V$ of the level set $\Omega=\left\{x \in R^{n} ; f(x)<f\left(x_{0}\right)\right\}$

(H2) The gradient $g_{k}$ is Lipschitz continuous in $V$, so a constant $\mathrm{M} \geq 0$ exists, such that

$$
\|g(x)-g(y)\| \leq M\|x-y\| \text { For all } x, y \in V
$$

The following lemma provides a lower bound for the steplength $t_{k}$ (generated by Algorithm 1). The result of this lemma will be needed in the rest of this section.

\subsection{Sufficient descent condition}

Theorem 1: suppose that the sequence $\left\{g_{k}\right\}$ and $\left\{d_{k}\right\}$ are generated by (2) (3) and FRA .the step length $t_{k}$, is determined by inexact line search (9) and (10) if $g_{k} \neq 0$, then $d_{k}$ possesses the sufficient descent condition: $g_{k}^{T} d_{k} \leq-C\left\|g_{k}\right\|^{2}$

Proof: By the formula (10), we have the following: $B_{k}^{F R A}=\lambda \frac{\left\|g_{k}\right\|^{2}}{\left\|g_{k-1}\right\|^{2}} \geq 0$

$$
\text { Hence we obtain } 0 \leq B_{k}^{F R A} \leq \frac{\left\|g_{k}\right\|^{2}}{\left\|g_{k-1}\right\|^{2}}
$$

Using (9) and (14), we get,

$$
\left|B_{k}^{F R A} g_{k}^{T} \cdot d_{k-1}\right| \leq \sigma \frac{\left\|g_{k}\right\|^{2}}{\left\|g_{k-1}\right\|^{2}}\left|g_{k}^{T} \cdot d_{k-1}\right|
$$

By (3), we have $d_{k}=-g_{k}+\beta_{k} d_{k-1}$

$$
\frac{g_{k}^{T} \cdot d_{k}}{\left\|g_{k}\right\|^{2}}=-1+B_{k}^{F R A} \frac{g_{k}^{T} d_{k-1}}{\left\|g_{k}\right\|^{2}}
$$

we have $g_{0}^{T} d_{0} \leq-\left\|g_{0}\right\|^{2}<0$

If $g_{0} \neq 0$; suppose that $\mathrm{d}_{\mathrm{i}} ; \mathrm{i}=1,2, \ldots, \mathrm{k}$; are all descente directions, that is $g_{k}^{T} d_{k}<0$

By (16); we get;

$$
\left|B_{k}^{F R A} g_{k}^{T} \cdot d_{k-1}\right| \leq-\sigma \frac{\left\|g_{k}\right\|^{2}}{\left\|g_{k-1}\right\|^{2}} g_{k}^{T} \cdot d_{k-1}
$$

That is;

$$
\sigma \frac{\left\|g_{k}\right\|^{2}}{\left\|g_{k-1}\right\|^{2}} g_{k}^{T} \cdot d_{k-1} \leq B_{k}^{F R A} g_{k}^{T} \cdot d_{k-1} \leq-\sigma \frac{\left\|g_{k}\right\|^{2}}{\left\|g_{k-1}\right\|^{2}} g_{k}^{T} \cdot d_{k-1}
$$


As shown in (17) and (18) deduce $-1+\sigma \frac{g_{k-1}^{T} \cdot d_{k-1}}{\left\|g_{k-1}\right\|^{2}} \leq \frac{g_{k}^{T} \cdot d_{k}}{\left\|g_{k}\right\|^{2}} \leq-1-\sigma \frac{g_{k-1}^{T} \cdot d_{k-1}}{\left\|g_{k-1}\right\|^{2}}$

By repeating this process and the fact $g_{0}^{T} d_{0}=-\left\|g_{0}\right\|^{2}$, we have

$$
-\sum_{i=0}^{k-1}(\sigma)^{i} \leq \frac{g_{k}^{T} d_{k}}{\left\|g_{k}\right\|^{2}} \leq-2+\sum_{i=0}^{k-1}(\sigma)^{i}
$$

As shown in (19). Can be written as;

$$
-\frac{1}{1-\sigma} \leq \frac{g_{k}^{T} d_{k}}{\left\|g_{k}\right\|^{2}} \leq-2+\frac{1}{1-\sigma}
$$

By making the restriction $\sigma \in(0,0.1)$ we have $g_{k}^{T} d_{k}<0$.

Now, we prove the sufficient descent condition of $d_{k}$ if $\sigma \in(0,1)$

Set $c=-2+\frac{1}{1-\sigma}$ then $0<c<1$, and (17) turns out to be;

$$
c-2 \leq \frac{g_{k}^{T} d_{k}}{\left\|g_{k}\right\|^{2}} \leq-c
$$

Thus we obtain $g_{k}^{T} d_{k} \leq-C\left\|g_{k}\right\|^{2}$ Or $\mathrm{C}=-2+\frac{1}{1-\sigma}$.

\subsection{Convergent analysis}

Lemma 1 Let the step length $t_{k}$ is generated by Algorithm 1 . Then, under the assumptions $\mathrm{H} 1$ and $\mathrm{H} 2$, there is a positive constant $\mathrm{C}$ such that,

$$
t_{k} \geq C \frac{\left\|g_{k}\right\|^{2}}{\left\|d_{k}\right\|^{2}}
$$

Proof: Subtracting $g_{k}^{T} d_{k}$ from both sides of (10) and using (19) we have

$$
-(1-\sigma) g_{k}^{T} d_{k} \leq\left(g_{k+1}-g_{k}\right)^{T} \leq M t_{k}\left\|d_{k}\right\|^{2}
$$

therefore;

$$
t_{k} \geq-\frac{(1-\sigma)}{M} \frac{g_{k}^{T} d_{k}}{\left\|d_{k}\right\|^{2}}
$$

with (10) we obtain:

$$
t_{k} \geq-\frac{(1-\sigma)}{M} \frac{\left\|g_{k}\right\|^{2}}{\left\|d_{k}\right\|^{2}}
$$

This inequality means that (25) satisfies with $C=-\frac{(1-\sigma)}{M}$, the proof is completed. The next lemma is known as Zoutendijk condition [24].

Lemma 2: Suppose assumption 1 hold and $d_{k}$ is generated by Algorithm 1, then;

$$
\sum_{n=0}^{\infty} \frac{\left\|g_{k}\right\|^{4}}{\left\|d_{k}\right\|^{2}}<\infty
$$

Proof: From (10) for any $k$ we have;

$$
f\left(x_{k}\right)-f\left(x_{k}+t_{k} d_{k}\right) \geq-\delta t_{k} g_{k}^{T} d_{k} \geq-\delta \frac{(1-\sigma)}{M} \frac{\left(g_{k}^{T} d_{k}\right)^{2}}{\left\|d_{k}\right\|^{2}}
$$

Moreover, from the hypothesis (1), we have that $\left\{f\left(x_{k}\right)\right\}$ is a decreasing sequence and has a limit in, which shows that $\lim _{k \rightarrow \infty} f\left(x_{k+1}\right)<+\infty$ and after (28) we have; 


$$
+\infty>f\left(x_{1}\right)-\lim _{k \rightarrow \infty} f\left(x_{k+1}\right) \geq \delta \frac{(1-\sigma)}{M} \sum \frac{\left(g_{k}^{T} d_{k}\right)^{2}}{\left\|d_{k}\right\|^{2}}
$$

Then $\sum \frac{\left(g_{k}^{T} d_{k}\right)^{2}}{\left\|d_{k}\right\|^{2}} \leq+\infty$, so, the proof is completed.

Theorem 2: we assume that $\mathrm{H} 1, \mathrm{H} 2$ hold, and the sequence $\left\{x_{k}\right\}$ is generated by the Algorithm 1, then, $\lim _{k \rightarrow \infty}\left\|\nabla f\left(x_{k}\right)\right\|=0$

\section{NUMERICAL EXPERIMENT}

In this part, we report numerical experiments that indicate the efficiency of the new algorithm. To this aim, we implement the new algorithm (Algorithm 1), Fletcher and Reeves (FR) algorithm and the modified Fletcher and Reeves (FR), WYL [10], DY [9], PRP [6]. The numerical results are given in the different initial points. We considered $\varepsilon=10^{-6}, \sigma=0.1, \delta=0.01$, under inexact line search of (SWP). We used MATLAB R2010 the performance results are shown in Figures 1-5 and compare their results obtained from solving of 17 test problems from [25].

In our experiments the stopping tolerance for the algorithms is Also, a failure is reported when $N I>20000$ or when the step length $t_{k}$ become less than eps $=10^{-6}$. We use we use the performance profiles in $[26,27]$. The total number of iterations, the total number of function evaluations, and the running time of each algorithm number of function evaluations. It can be seen that the FRA is the best solver with probability around $80 \%$, while the probability of solving a problem as the best solver is around $60 \%, 26 \%, 18 \%$ and $7 \%$ for the FR, PRP, WYL and the DAY respectively. The performance index in. Figure 2 is the total number of iterations. From this figure, we observe that the NEW method (FRA) obtains the most wins on approximately $70 \%$ of all test problems an the probability of being best solver is $55 \%, 29 \%, 26 \%$ and $8 \%$ for the FR, PRP, WYL and the DAY respectively.

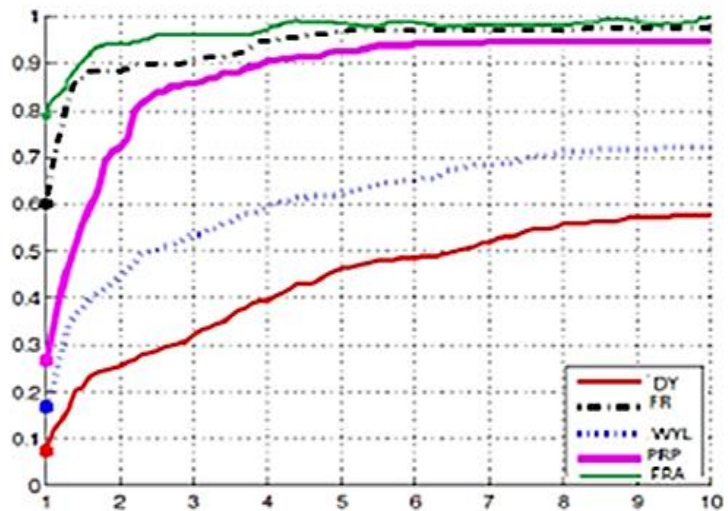

Figure 1. Performance profiles based on the number of function evaluations

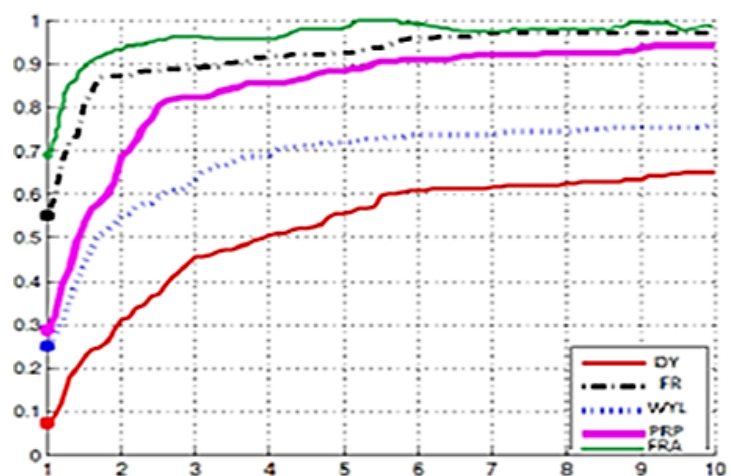

Figure 2. Performance of the number of iterations

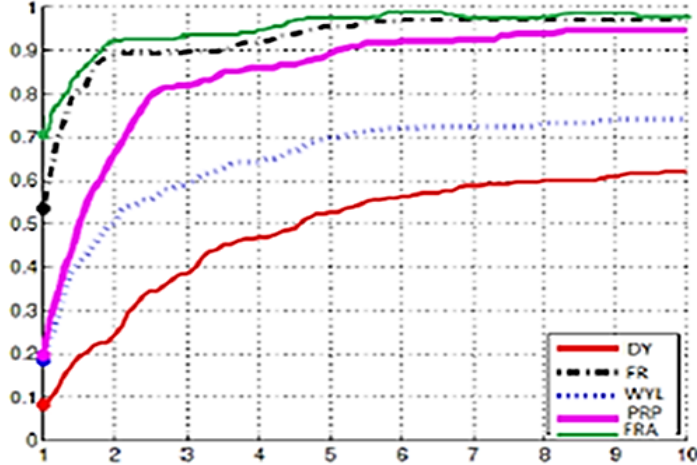

Figure 3. Performance profiles for running times 
The CPU time is illustrated in Figure 3. From this figure, it can be observed that the NEW is the best algorithm. Another important factor of these three figures is that the graph of the NEW algorithm grows up faster than the other algorithms. From the presented results, we can observe that the FRA method is best than the FR, PRP, WYL and the DAY methods. In solving unconstrained optimization problems.

Example 1: Extended Rosenbrock function $\left(x_{1}, x_{2}, \ldots x_{n}\right)=\sum_{i}^{n-1}\left[100\left(x_{i-1}-x_{i}^{2}\right)^{2}+\left(x_{i}-1\right)^{2}\right], n=2$, $x$ optimal $=(1,1)$

In Table 1, The FRA method was successful in all attempts to achieve the optimal solution, while the other methods failed.

- Remark 1: Table 2, shows that "FRA" has the best results since it solves about 100\% from the test problems. Figures 4 and 5 list the comparison of FR method and DY, WYL, PRP, FR methods x0 = [1 7]

Table 1. Numerical results for FRA, FR, PRP, WYL and DYin terms ofnumber iterations (NI) and CPU time with the strong wolf condition $\varepsilon=10^{-6} ; \sigma=0.1 ; \delta=0.01 ; \kappa=0.9$

\begin{tabular}{cccccc}
\hline Initial point & FRA & FR & PRP & WYL & DY \\
\hline & NI/CPU & NI/CPU & NI/CPU & NI/CPU & NI/CPU \\
$(10000,10000)$ & $637 / 6.41$ & Failed & Failed & Failed & Failed \\
$(100000,100000)$ & $934 / 5.74$ & Failed & Failed & Faled & Failed \\
$(1000,1000)$ & $299 / 0.887$ & Failed & Failed & Failed & Failed \\
$(-1,3)$ & $196 / 0.600$ & $313 / 2.528$ & $4.66 / 2.80$ & $14532 / 78.11$ & $170 / 2.266$ \\
$(100,100)$ & $161 / 1.68$ & $4693 / 20.043$ & Failed & Failed & Failed \\
$(1,3)$ & $122 / 0.414$ & $104 / / 4.019$ & $243 / 1.43$ & Failed & $112 / / 0.23$ \\
$(0,-9)$ & $163 / 0.533$ & $355 / 0.89$ & $340 / 1.639$ & Failed & Failed \\
$(1,7)$ & $67 / 0.533$ & $230 / 2.541$ & $737 / 5.739$ & $4470 / 15.315$ & $149 / 1.195$ \\
\hline
\end{tabular}

Table 2. Comparing the results obtained in Table 1

\begin{tabular}{ccc}
\hline Méthod & Ranking & The success rate \\
\hline FRA & 1 & $100 \%$ \\
FR & 2 & $55 \%$ \\
PRP & 3 & $44 \%$ \\
DY & 4 & $33 \%$ \\
WYL & 5 & $22 \%$ \\
\hline
\end{tabular}

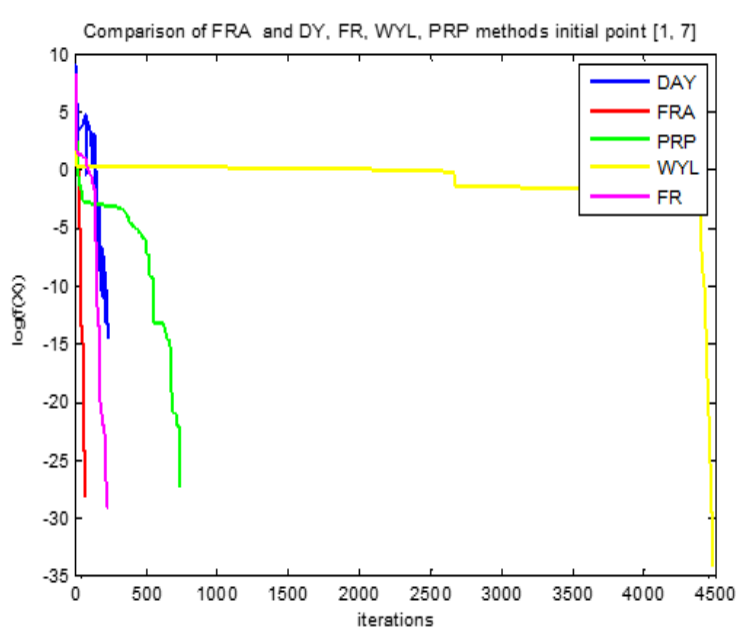

Figure 4. Performance of the number of function evaluations

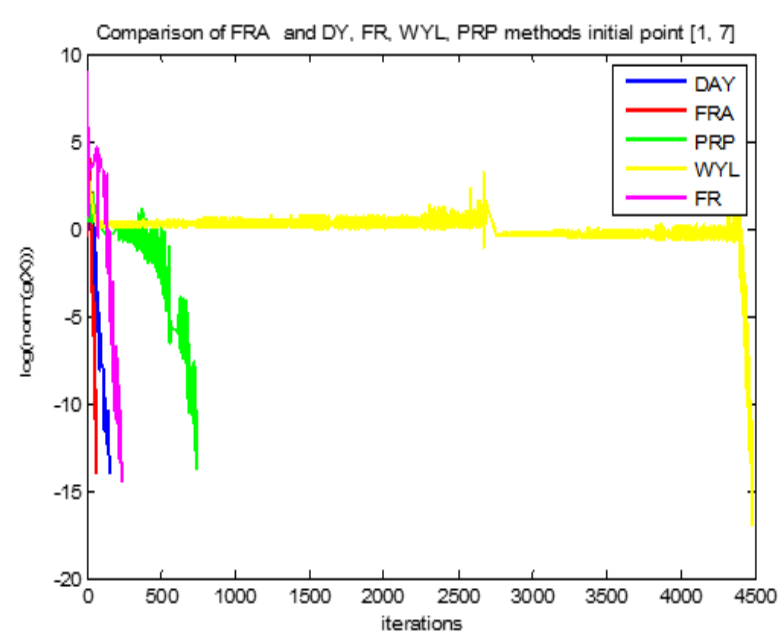

Figure 5. Performance of the number o f gradient evaluations

- Remark 2: From the Figures 4 and 5, The FRA method performs better than other methods by selecting a starting point with the Resenbrock function $f\left(x_{1}, x_{2}, \ldots x_{n}\right)=\sum_{i}^{n-1}\left[100\left(x_{i-1}-x_{i}^{2}\right)^{2}+\left(x_{i}-1\right)^{2}\right]$, $n=2$. And she is best performance in terms of values gradients and functions and the number of iterations 


\section{CONCLUSION}

in this paper, we have proposed a new CGmethod named FRA for solving a large-scale unconstrained optimization problem. We proved the global convergence of this method and sufficient descent condition under the inexact line search of (SWP) numerical experiment show that the new method FRA is more efficient than the others methods DAY, WYL, FR, and PRP.

\section{ACKNOWLEDGEMENTS}

I would like to thank the anonymous rulers and the editor for their accurate and valuable reading. Suggestions that led to a correction of errors and a significant improvement in the paper.

\section{REFERENCES}

[1] A. Pietrabissa and L. Ricciardi Celsi, "Discrete-Time Selfish Routing Converging to the Wardrop Equilibrium," in IEEE Transactions on Automatic Control, vol. 64, no. 3, pp. 1288-1294, 2019.

[2] A. Di Giorgio, et al., "On the optimization of energy storage system placement for protecting power transmission grids against dynamic load altering attacks," 2017 25th Mediterranean Conference on Control and Automation (MED), Valletta, 2017, pp. 986-992.

[3] L. R. Celsi, et al., "On the many-to-many carpooling problem in the context of multi-modal trip planning," 2017 25th Mediterranean Conference on Control and Automation (MED), Valletta, 2017, pp. 303-309.

[4] V. Suraci, L. R. Celsi, A. Giuseppi and A. Di Giorgio, "A distributed wardrop control algorithm for load balancing in smart grids," 2017 25th Mediterranean Conference on Control and Automation (MED), Valletta, 2017, pp. 761-767.

[5] R. Fletcher and C. M. Reeves, "Function minimization by conjugate gradients," The Computer Journal, vol. 7, pp. 149-154, 1964.

[6] B. T. Polyak, "The conjugate gradient method in extremem problems," USSR Computational Mathematics and Mathematical Physics, vol. 9, no. 4, pp. 94-112, 1969.

[7] Dai, Y., "Convergence of nonlinear methods," Gournal of Computational Mathematics, vol. 19, pp. 539-549, 2001.

[8] M. R. Hestenes and E. Stiefel, "Methods of conjugate gradients for solving linear systems," Journal of Research of the National Bureau of Standards, vol. 49, no. 6, pp. 409-436, 1952.

[9] Y. H. Dai and Y. Yuan, "A nonlinear conjugate gradient method with a strongglobal convergence property," SIAM Journal on Optimization, vol. 10, no. 1, pp. 177-182, 1999.

[10] Weiet, et al., "The convergence properties of some conjugate gradient methods," Applied Mathematics and Computation, vol. 183, pp. 1341-1350, 2006.

[11] Dai, Y. and Yuan, Y., "Nonlinear conjugate gradient methods," shanghai scientic, 2000.

[12] Gilbert, J. C. and Nocedal, J., "Global convergence properties of conjugate gradient methods for optimization," SIAM Journal on optimization, vol. 2, no. 1, pp. 21-42, 1992.

[13] Guanghui, L., Jiye, H., and Hongxia, Y., "Global convergence of the. fletcher-reeves algorithm with inexact linesearch," Applied Mathematics- A Journal of Chinese Universities, vol. 10, no. 1, pp. 75-82, 1995.

[14] Hager, W. W. and Zhang, H., "A survey of nonlinear conjugate gradient methods," Pacic journal of Optimization, vol. 2, no. 1, pp. 35-58, 2006.

[15] Liu, H., Wang, H., Qian, X., and Rao, F., "A conjugate gradient method with sufficient descent property," Numerical Algorithms, vol. 70, no. 2, pp. 269-286, 2015.

[16] Livieris, I. E., Tampakas, V., and Pintelas, P., "A descent hybrid conjugates gradient method based on the memorylessbfgs update," Numerical Algorithms, vol. 79, no. 4, pp. 1169-1185, 2018.

[17] Yao, S., He, D., and Shi, L., "An improved perry conjugate gradient method with adaptive parameter choice," Numerical Algorithms, vol. 78, no. 3, pp. 1-15, 2018.

[18] Al-Baali, M., "Descent property and global convergence of the. Fletcher-reeves method with inexact line search," IMA Journal of Numerical Analysis, vol. 5, no. 1, pp. 121-124, 1985.

[19] Hager, W. W. and Zhang, H., "Algorithm 851: Cg descent, a conjugate gradient method with guaranteed descent," ACM Transactions on Mathematical Software (TOMS), vol. 32, no. 1, pp. 113-137, 2006.

[20] Zhang, L., Zhou, W., and Li, D., "Global convergence of a modified. Fletcher-reeves conjugate gradient method with Armijo-type line search," Numerische Mathematik, vol. 104, no. 4, pp. 561-572, 2006

[21] Nocedal, J. and Wright, S., "Numerical optimization," Springer Science \& Business Media, 2006.

[22] S. Sanmtias and E. Vercher E., "A generalized conjugate gradient algorithm," Journal of Optimization Theory and Applications, vol. 98, pp. 489-502, 1998.

[23] Z. J. Shi, "Nonlinear conjugate gradient method with exact line search (in Chinese)," Acta Mathematica Sinica, English Series, vol. 24, no. 6, pp. 675-682, 2004.

[24] G. Zoutendijk, "Nonlinear programming computational methods," in J. Abadie (Ed.), Integer and Nonlinear Programming, North-Holland, Amsterdam, pp. 37.86, 1970.

[25] Gould, N. I., Orban, D., and Toint, P. L., "Cutest: a constrained and unconstrained testing environment with safe threads for mathematical optimization," Computational Optimization and Applications, vol. 60, no. 3, pp. 545-557, 2015.

[26] Dolan, E. D. and More, J. J., "Benchmarking optimization software with performance profiles," Mathematical Programming, vol. 91, no. 2, pp. 201-213, 2002.

[27] N. Andrei, "An unconstrained optimization test functions ollection," Advanced Modeling and Optimization, vol. 10, pp. 147-161, 2008. 